\title{
Efecto de la temperatura de pasteurización y proporción de mezclas binarias de pulpa de carambola y mango sobre su capacidad antioxidante lipofílica
}

\author{
Christian René Encina Zelada ${ }^{1}$ \\ Andrea Paola Bernal Sánchez \\ Daniel Rojas Hurtado \\ Universidad Nacional Agraria La Molina. Lima, Perú \\ Recibido 1/5/2013/ Aprobado 21/6/2013
}

REsumen: El efecto tipo parábola de la temperatura de pasteurización y de la proporción de mezclas binarias de pulpa de carambola y mango sobre la capacidad antioxidante lipofílica fueron estadísticamente significativos (valor p menor de 0,05) para ambos casos. La maximización de la capacidad antioxidante lipofílica aplicando la metodología de Superficie de Respuesta fue para el factor mezclas binarias de mango:carambola de la proporción de 51:49; y para el factor temperatura de pasteurización, el valor de $91^{\circ} \mathrm{C}$, obteniéndose un valor de $0,333 \mu \mathrm{mol}$ eq Trolox/g.

Palabras clave: Mango / carambola / superficie de respuesta / capacidad antioxidante lipofílica / frutas / Perú

\section{Effect of pasteurization temperature and binary mixtures of pulp from carambola and mango on its lipophilic antioxidant capacity}

ABSTRACT: The parable rate effect of pasteurization and the proportion of binary mixtures of pulp from carambola and mango on its lipophilic antioxidant capacity were statistically significant (p value less than 0.05 ) for both cases. Maximization of lipophilic antioxidant capacity using the methodology response surface were for factor binary mixtures the proportion mango:carambola of 51:49, and for the pasteurization the value of $91^{\circ} \mathrm{C}$, obtaining a value of $0.333 \mu \mathrm{mol}$ eq Trolox/g.

Keywords: Mango / starfruit / response surface / lipophilic antioxidant capacity / fruits / Peru

1 Correos electrónicos: cencina@lamolina.edu.pe, sapabea@hotmail.com, danielrojashp@hotmail.com

Ingeniería Industrial n. ${ }^{\circ} 31$, enero-diciembre 2013, ISSN 1025-9929, pp. 197-219 


\section{INTRODUCCIÓN}

El mango (Mangifera indica L.) es una especie arbórea, tropical, nativa del sudeste asiático y originado en los montes Himalaya, de la India y la parte oeste de Birmania. En América Latina se considera que el primer país en cultivar el mango fue Brasil. La fruta del mango está constituida por 3 partes básicas: cáscara, pulpa y semilla. El porcentaje en peso de estas partes varía según la variedad de mango y según las condiciones climáticas y agronómicas, aunque en promedio dichas partes vendrían a constituir un 45,5-82,9\% de pulpa, un 9,1-26,8\% de semilla y un 7,1-39,2\% de cáscara (Ottone, 2001). El mango se caracteriza por ser una fuente importante de vitaminas A y B, y contiene variantes de vitamina C (Purseglove, 1974; citado por Milacatl, 2003). El contenido de ácido ascórbico y la acidez total disminuyen durante el desarrollo del fruto, mientras que los carotenoides y azúcares totales aumentan (Laskshminarayana, 1973; citado por Milacatl, 2003).

Según Calzada (1980), la carambola (Averrhoa carambola) es originaria de Asia tropical, más específicamente de la India o Indonesia. En el Perú fue introducida desde Brasil vía la Amazonía por viajeros que hacían esa ruta; luego se extendió a los departamentos de Huánuco, Madre de Dios y el Cusco. Por otro lado, Andrade-Cuvi et al. (2010) señalan que es conocida como fruta estrella o starfruit, tiene forma ovalada y alargada, con cinco aristas o alas, y al cortarse toma la forma de estrella de cinco puntas. Es de tamaño pequeño, con una longitud que oscila entre 7 y $12 \mathrm{~cm}$, con piel fina, lustrosa y comestible, de color entre verde o dorado y amarillo-anaranjado cuando está madura. La pulpa es crujiente, de suave textura y amarilla vidriosa; es abundante, jugosa y con un fino sabor agridulce, y tiene pocas o ninguna semilla.

Umme (1996), citado por Amaro et al. (2002), señala que la población microbiológica de pulpa de frutas ácidas es relativamente restringida y consiste usualmente de mohos y levaduras, bacterias acéticas y lácticas, que generalmente son sensibles al calor. Los tratamientos térmicos de estas pulpas son realizados a temperatura generalmente menor a $100{ }^{\circ} \mathrm{C}$, ya que los microorganismos tienen una baja resistencia térmica. Otros microorganismos son usualmente inhibidos por la acidez de las pulpas de frutas. El tipo de microorganismos presente en un alimento depende principalmente del $\mathrm{pH}$ y la actividad del agua. En los alimentos ácidos, como los zumos o purés de fruta $(\mathrm{pH}$ menor o igual a 4,5), los microorganismos que crecen se controlan fácilmente con un tratamiento térmico de pasteurización suave (Osorio, 2008). 
Respecto a los microorganismos importantes en el tratamiento térmico de alta acidez, como frutas con valor de $\mathrm{pH}$ menor a 4, estos son: Byssochlamys fulva, Byssochlamys nívea y Lactobacillus sp (Alvarado, 2011). Byssochalmys es el género más resistente y común causante del deterioro en productos de frutos y esto se debe a la resistencia de sus ascoesporas y a su amplia distribución en el ambiente (Beauchat \& Rice, 1981, citado por Gómez, 2004).

Un antioxidante es una molécula capaz de retardar o prevenir la oxidación de otras moléculas. El sistema antioxidante de defensa está constituido por compuestos de naturaleza enzimática como el superóxido dismutasa, la catalasa, la glutatión peroxidasa y los compuestos de naturaleza no enzimática, entre estos la vitamina E, el B-caroteno, la vitamina $\mathrm{C}$, el glutatión reducido, la albúmina, los flavonoides y los metales de transición como selenio, cobre y zinc, entre otros (Castañeda et al., 2008).

Neira y Yuri (2004) mencionan que algunos de los antioxidantes son vitaminas, tales como la vitamina $\mathrm{A}, \mathrm{C}$ y E; otros son flavonoides (quercetina, catequina), antocianinas, carotenoides o ácidos fenólicos (ácido caféico y ácido clorogénico). El poder antioxidante de algunos compuestos ha sido clasificado de menor a mayor grado de la siguiente forma: Vitamina $\mathrm{C}(=4)$, B-caroteno $(=5)$, vitamina $\mathrm{E}(=6)$, frutas y verduras (=8).

La pasteurización, proceso térmico creado por Pasteur en 1864, tiene como objetivo el exterminio parcial de la flora banal y la eliminación total de la flora microbiana patógena, además de inactivar enzimas perjudiciales. Es un tratamiento térmico relativamente suave (temperaturas generalmente inferiores a $100{ }^{\circ} \mathrm{C}$ ), que se utiliza para prolongar la vida útil de los alimentos durante varios días o meses (Fellows, 2000).

El calentamiento de los alimentos se puede llevar a cabo con vapor, con agua caliente, con calor seco, o con corrientes eléctricas, y se enfrían inmediatamente después de haber sido sometidos al tratamiento térmico (Frazier, 1993).

Además, es comúnmente aceptado que las frutas y por ende las pulpas contengan cantidades de vitaminas y provitaminas antioxidantes, fenoles y polifenoles antioxidantes, ácido ascórbico, tocoferoles, carotenoides, flavonoides, etcétera, que son beneficiosas para la salud y, en este caso en particular, queremos darle un enfoque tecnológico al 
obtener una pulpa de mango y carambola, que, además de darle un valor agregado a esa materia prima, sirve para difundir sus compuestos bioactivos en productos tan masivos como la pulpa (Encina, 2006).

Con esta información, se estableció como objetivo principal de la investigación determinar el efecto de la temperatura de pasteurización y de la proporción de mezclas binarias de pulpa de carambola (Averrhoa carambola) y mango (Mangifera indica L.) sobre su capacidad antioxidante lipofílica.

\section{PARTE EXPERIMENTAL}

\subsection{Lugar de ejecución}

El presente trabajo de investigación se realizó en los laboratorios de Fisicoquímica, Microbiología y Planta Piloto de Alimentos, de la Facultad de Industrias Alimentarias de la Universidad Nacional Agraria La Molina.

\subsection{Materiales y reactivos}

Muestras: Carambola (Averrhoa carambola) y Mango (Mangifera indi$c a$ L.) variedad Haden.

Reactivos: ABTS (2,2'-azino-bis(3-thylbenzthiozoline-6-sulphonic) acid) adquirido de Sigma Chemical Co. ${ }^{\circledR}$, persulfato de potasio de grado analítico de Harleco $7574^{\circledR}$, etanol absoluto y hexano de J.T. Baker ${ }^{\circledR}$, metanol de Mallinckrodt ${ }^{\circledR}$, acetona, folin-ciocalteu, carbonato de sodio anhidro al 1 N, 2,6 diclorofenol indofenol de Ferak $33095^{\circledR}$, ácido metafósforico, ácido acético glacial 100\% de $\mathrm{Merck}^{\circledR}$, ácido cítrico anhidro extra puro de $\mathrm{Schardau}^{\circledR}$ y alcohol de $96^{\circ} \mathrm{GL}$.

\subsection{Métodos de análisis}

- Análisis proximal: humedad, cenizas totales, lípidos, proteína total, fibra cruda, carbohidratos (AOAC 1990).

- Análisis fisicoquímicos: pH, acidez titulable y contenido de sólidos solubles (AOAC, 1990).

- Capacidad antioxidante: medida por los métodos ABTS (Re et al., 1999). 


\subsection{Metodología experimental}

- Etapa 1: Caracterización de la materia prima

Se determinó la composición fisicoquímica mediante el análisis proximal, $\mathrm{pH}$, sólidos solubles y acidez total. También se determinó la capacidad antioxidante lipofílica de las materias primas. Asimismo, se realizó un análisis microbiológico a las materias primas antes de iniciar el procesamiento y el tratamiento térmico. Para este fin se realizó el cultivo en medio OGA para recuento de mohos y levaduras según las especificaciones de la ICMSF (2000).

- Etapa 2: Determinación del tiempo de tratamiento térmico

$\mathrm{Al}$ ser las pulpas binarias de mango y carambola productos con $\mathrm{pH}$ menor de 4,5, se consideró el Bysosschlamys fulva como microorganismo de referencia con un valor $\mathrm{D}_{93,3^{\circ} \mathrm{C}}$ de un minuto y un valor $\mathrm{Z}$ $=8,9^{\circ} \mathrm{C}$. Se calculó el tiempo de tratamiento térmico necesario para reducir la población inicial entre 3D a 5D (Ranganna, 1977; citado por Encina, 2005). Para el cálculo del tiempo de tratamiento térmico equivalente para las temperaturas de 85,90 y $95{ }^{\circ} \mathrm{C}$ se usaron las siguientes ecuaciones:

$$
\mathrm{UP}=\mathrm{P}_{0}=\mathrm{D}_{0} \times(\log \mathrm{a}-\mathrm{b})
$$

Donde:

$\mathrm{P}_{\mathrm{o}}=\mathrm{UP}$ = es la suma de todos los efectos letales considerados a 93,3 ${ }^{\circ} \mathrm{C}$ expresados en minutos.

$\mathrm{D}_{\mathrm{o}}=$ valor de $\mathrm{D}$ a la temperatura de referencia de $93,3{ }^{\circ} \mathrm{C}$ para Bysosschlamys fulva.

$\mathrm{a}=$ número inicial de microorganismos antes del tratamiento térmico, el cual se determinó con el recuento en medio microbiológico OGA.

$\mathrm{b}$ = número final de microorganismos esperado después del tratamiento térmico a una temperatura constante.

$$
\left.\mathrm{Px}=\mathrm{P}_{0} \times 10^{((\mathrm{T} 0-\mathrm{Tx}) / \mathrm{z})}\right)
$$

$\mathrm{P}_{\mathrm{x}}=$ tiempo de calentamiento a otra temperatura equivalente en minutos a la temperatura de referencia

$\mathrm{P}_{\mathrm{o}}=$ tiempo requerido para destruir un número específico de microorganismos a $93,3{ }^{\circ} \mathrm{C}$

$\mathrm{T}_{\mathrm{o}}=$ temperatura de referencia, $93,3{ }^{\circ} \mathrm{C}$ 
$\mathrm{T}_{\mathrm{x}}=$ temperatura a evaluar en el medio de calentamiento para realizar la pasteurización

$\mathrm{Z}=8,9^{\circ} \mathrm{C}$, parámetro cinético del Bysosschlamys fulva

- Etapa 3: Determinación del efecto de la temperatura de pasteurización y de la proporción de las mezclas binarias para maximizar su capacidad antioxidante lipofílica

En el tratamiento térmico se determinó el efecto de las diferentes temperaturas de pasteurización y en las mezclas binarias se determinó el efecto de las diferentes mezclas sobre la capacidad antioxidante lipofílica. Adicionalmente, en esta etapa se realizó la determinación de la superficie de respuesta de los dos factores antes mencionados, para maximizar de esta forma la capacidad antioxidante lipofílica.

- Etapa 4: Caracterización del producto final

Para analizar el producto final «pulpa de mango y carambola que maximiza su capacidad antioxidante lipofílica», se realizaron los siguientes análisis: fisicoquímico y proximal, $\mathrm{pH}$, sólidos solubles, acidez titulable, análisis químico y análisis microbiológico.

\subsubsection{Análisis estadístico}

Los resultados están expresados como el valor promedio \pm desviación estándar de tres réplicas. En la etapa 3 se aplicó la metodología de Superficie de Respuesta, trabajando con un arreglo factorial $3^{\mathrm{n}}$ aplicado a los factores de temperatura de pasteurización y de la proporción de las mezclas binarias de pulpa de frutas, con lo cual se obtuvo la máxima capacidad antioxidante lipofílica. Para el tratamiento de los resultados experimentales en esta etapa se utilizó una copia de evaluación del paquete estadístico Statgraphic Centurion XVIâ (StatPoint, 2013).

\section{RESULTADOS Y DISCUSIÓN}

\subsection{Caracterización de la materia prima}

\subsubsection{Análisis proximal y fisicoquímico}

En las tablas 1 y 2 se muestran los resultados del análisis fisicoquímico de la pulpa de mango y carambola. Se observa que en ambos casos la humedad hallada es superior a los reportados por Reyes et al. (2009); estas diferencias de resultados pueden deberse a los diferentes estados 
de madurez de las materias primas. El contenido de cenizas hallado en el caso del mango es similar a los datos reportados por Reyes et al. (2009) y para la carambola inferiores a estos.

Tabla 1

Análisis fisicoquímico del mango (Mangifera indica L.) variedad Haden

\begin{tabular}{lc}
\hline \multicolumn{1}{c}{ Componentes } & Contenido $(\mathbf{g} / \mathbf{1 0 0} \mathbf{g})$ \\
\hline Humedad (\%) & $83,21 \pm 0,01$ \\
Cenizas totales (\%) & $0,52 \pm 0,01$ \\
Grasa (\%) & $0.53 \pm 0,02$ \\
Proteína (\%) & $0,57 \pm 0,01$ \\
Fibra cruda (\%) & $0,48 \pm 0,02$ \\
Carbohidratos (\%) & $15,17 \pm 0,01$ \\
Acidez total (g ácido cítrico/ $100 \mathrm{ml}$ fruto) & $0,51 \pm 0,01$ \\
pH & $4,32 \pm 0,02$ \\
Sólidos solubles & $14,5 \pm 0,4$ \\
Índice de madurez & $28,21 \pm 0,34$ \\
\hline
\end{tabular}

Elaboración propia.

El contenido de proteínas para el mango es ligeramente mayor y para carambola es inferior a lo reportado por Reyes et al. (2009).

Tabla 2

Análisis fisicoquímico de la carambola (Averrhoa carambola)

\begin{tabular}{lc}
\hline \multicolumn{1}{c}{ Componentes } & Contenido (g/100 g) \\
\hline Humedad (\%) & $94,54 \pm 0,02$ \\
Cenizas totales (\%) & $0,24 \pm 0,01$ \\
Grasa (\%) & $0,16 \pm 0,01$ \\
Proteína (\%) & $0,29 \pm 0,01$ \\
Fibra cruda (\%) & $0,31 \pm 0,02$ \\
Carbohidratos (\%) & $4,77 \pm 0,01$ \\
Acidez total (g ácido cítrico/100 ml fruto) & $1,93 \pm 0,08$ \\
pH & $1,66 \pm 0,02$ \\
Sólidos solubles & $6,3 \pm 0,4$ \\
Índice de madurez & $3,27 \pm 0,13$ \\
\hline
\end{tabular}

Elaboración propia. 
La variación de los resultados de los diferentes autores citados se podría atribuir a la proveniencia y condiciones ambientales de cada muestra. En la mayoría de casos la fruta contiene una cantidad de agua significativa, de modo que se considera de bajo valor nutritivo; básicamente como un producto fruitivo y de consumo.

Respecto al índice de madurez, para ambas materias primas se trabajó con estadíos de madurez intermedia, y para hallar un valor objetivo se realizó la determinación de los sólidos solubles y de la acidez. La división de ambas da como resultado el índice de madurez. Durante el proceso de maduración existe una serie de enzimas que reaccionan con sus sustratos en la fruta: el almidón se descompone en glucosa, que en parte se isomeriza en forma de fructosa, por lo que el contenido de sólidos solubles va aumentando conforme la fruta va madurando (Baltes, 2007).

\subsubsection{Determinación de la capacidad antioxidante lipofílica}

Se determinó la actividad antioxidante lipofílica de las pulpas de mango y carambola por el método ABTS, que según Mesa-Vanegas et al. (2010) se fundamenta en la cuantificación de la decoloración del radical ABTS $^{\cdot+}$, debido a la interacción con especies donantes de hidrógeno o de electrones. El radical catiónico $\mathrm{ABTS}^{++}$es un cromóforo que absorbe a una longitud de onda de $734 \mathrm{~nm}$ y se genera por una reacción de oxidación del ABTS (2,2'-azino-bis- [3-etil benzotiazolin-6-sulfonato de amonio]) con persulfato de potasio. Las mediciones se realizaron a una longitud de onda de $734 \mathrm{~nm}$. En la evaluación se utilizaron 10 microlitros de extracto y 990 microlitros de la solución del radical $\mathrm{ABTS}^{\cdot+}$. A los 30 minutos de reacción a temperatura ambiente y en la oscuridad se leyó el cambio en la absorbancia respecto a la referencia del reactivo, a una longitud de onda de $734 \mathrm{~nm}$. La referencia del reactivo consistió en una solución del radical $\mathrm{ABTS}^{\cdot+}$ con el solvente de la muestra. Los resultados se expresaron como valores TEAC (Trolox equivalent antioxidant capacity) mediante la construcción de una curva patrón, usando como antioxidante Trolox. Los resultados se muestran en la tabla 3. 
Tabla 3

Capacidad antioxidante lipofílica de las pulpas de mango (Mangifera indica L.) y carambola (Averrhoa carambola)

\begin{tabular}{lc}
\hline \multicolumn{1}{c}{ Pulpa } & $\begin{array}{c}\text { capacidad antioxidante lipofílica } \\
(\boldsymbol{\mu m o l} \text { eq Trolox/g) }\end{array}$ \\
\hline Mango & $0,360 \pm 0,02$ \\
Carambola & $0,320 \pm 0,02$ \\
\hline
\end{tabular}

Elaboración propia.

La capacidad antioxidante de un alimento depende de la naturaleza y concentración de los compuestos bioactivos presentes en él. El mango y la carambola, a pesar de tener grandes cantidades de carotenoides, tienen una capacidad antioxidante relativamente baja, esto debido a que los carotenoides son eficientes en la extinción (quenching) de singletes de oxígeno y no tanto en el atrapamiento de radicales peroxílicos (Pineda et al., 1999; citado por Encina 2006).

La evaluación capacidad antioxidante mediante el método del ABTS se efectuó utilizando una mezcla de isopropanol/hexano, es decir se cuantificó la capacidad antioxidante de compuestos lipofílicos (carotenoides).

\subsubsection{Análisis microbiológico}

Como se observa en la tabla 4, las pulpas de mango y carambola contaban con un bajo contenido de unidades formadoras de colonia (u.f.c.). Estos valores son relativamente bajos, especialmente los de hongos y levaduras, debido a que el mango es una fruta con una cubierta gruesa. El bajo contenido de hongos y levaduras (100 u.f.c./g) también se puede deber al bajo $\mathrm{pH}$ que contienen el mango $(4,32)$ y la carambola $(1,66)$, ya que todos los hongos y levaduras crecen bien a valores de $\mathrm{pH} 5 \mathrm{o}$ mayores (ICMSF, 2000).

Tabla 4

Carga microbiana de mohos y levaduras en las pulpas de mango (Mangifera indica L.) y carambola (Averrhoa carambola)

\begin{tabular}{lc}
\hline \multicolumn{1}{c}{ Pulpa } & $\begin{array}{c}\text { Mohos y levaduras } \\
\text { (u.f.c./ml) }\end{array}$ \\
\hline Mango & $2,0 \times 10^{4}$ u.f.c./g \\
Carambola & $7,5 \times 10^{4}$ u.f.c./g \\
\hline
\end{tabular}

Elaboración propia. 


\subsection{Determinación del tiempo de tratamiento térmico}

Rees y Bettison (1994) mencionan que un alimento «estéril comercialmente» puede definirse como un producto que ha sido sometido a un tratamiento térmico tal que no se altera en condiciones normales de almacenamiento ni supondrá un peligro para la salud del consumidor. Por ejemplo, un producto ácido, como una fruta, puede haber sido sometido a un proceso de pasteurización suficiente para acabar con levaduras, mohos y bacterias no esporuladas, aunque insuficiente para destruir los esporos bacterianos. Con la excepción de algunas especies acidúricas, la presencia de esporos bacterianos en productos muy ácidos se considera como un hecho sin importancia, ya que la acidez evita su desarrollo.

En primer lugar se determinó el valor de pasteurización «P» requerido o, en este caso, también llamado valor «UP», para el proceso de pasteurización. Se calculó una carga inicial de 7,5 x $10^{4}$ u.f.c. $/ \mathrm{ml}$, la cual se determinó mediante la realización de pruebas microbiológicas. Del mismo modo se tomó en cuenta el recuento de hongos y levaduras, pues estos atacan con mayor frecuencia el fruto. Ranganna (1977), citado por Encina (2005), afirma que para alimentos con $\mathrm{pH}$ menor de 4,5 se requiere un nivel de reducción de 3 a 5 ciclos logarítmicos. Basándonos en esa premisa se define un valor UP de 4 para llegar a un nivel intermedio en cuanto a la intensidad del tratamiento térmico, con lo cual alcanzaríamos una reducción de la carga microbiana a valores menores de 1 u.f.c./ml.

Los cálculos para la determinación del tiempo de pasteurización para cada temperatura definida se muestran resumidamente en la tabla 5, y de manera detallada se pueden apreciar en el anexo 1.

Tabla 5

Tiempo de tratamiento térmico calculado para las mezclas binarias de mango:carambola

\begin{tabular}{cc}
\hline $\begin{array}{c}\text { Temperatura } \\
\text { de pasteurización }\left({ }^{\circ} \mathrm{C}\right)\end{array}$ & $\begin{array}{c}\text { Tiempo de pasteurización } \\
\text { (minutos) }\end{array}$ \\
\hline 85 & 16,02 \\
90 & 1,90 \\
95 & 0,24 \\
\hline
\end{tabular}

Elaboración propia. 
Estos datos nos permitieron tener tiempos y temperaturas equivalentes, es decir que causan la misma disminución de la carga microbiana inicial. A partir de ellos se elaboraron las muestras de pulpas de mango:carambola que fueron evaluadas para la determinación de la capacidad antioxidante lipofílica y su posterior maximización.

\subsection{Determinación del efecto de la temperatura de pasteurización y de la proporción de las mezclas binarias para maximizar su capacidad antioxidante lipofílica}

En la tabla 6 se presentan los dos factores y sus niveles ensayados para la maximización de la capacidad antioxidante lipofílica mediante el método de Superficie de Respuesta, teniendo como primer factor diferentes proporciones de pulpa de mango y carambola, y como segundo factor la temperatura de pasteurización de las pulpas antes mencionadas, que se sometieron a la cuantificación de su capacidad antioxidante en la fase lipofílica determinada como $\mu \mathrm{mol}$ eq Trolox/g de muestra, después de su procesamiento.

Tabla 6

Capacidad antioxidante de las pulpas binarias pasteurizadas

\begin{tabular}{ccc}
\hline $\begin{array}{c}\text { Proporción } \\
\text { (mango:carambola) }\end{array}$ & $\begin{array}{c}\text { Temperatura de } \\
\text { pasteurización }\left({ }^{\circ} \mathbf{C}\right)\end{array}$ & $\begin{array}{c}\text { Capacidad antioxidante lipofílica } \\
(\boldsymbol{\mu m o l ~ e q ~ T r o l o x / g ) ~}\end{array}$ \\
\hline $40: 60$ & 85 & $0,21 \pm 0,03$ \\
$40: 60$ & 90 & $0,23 \pm 0,03$ \\
$40: 60$ & 95 & $0,28 \pm 0,03$ \\
$50: 50$ & 85 & $0,23 \pm 0,02$ \\
$50: 50$ & 90 & $0,34 \pm 0,01$ \\
$50: 50$ & 95 & $0,27 \pm 0,03$ \\
$60: 40$ & 85 & $0,24 \pm 0,02$ \\
$60: 40$ & 90 & $0,34 \pm 0,01$ \\
$60: 40$ & 95 & $0,21 \pm 0,04$ \\
\hline
\end{tabular}

Elaboración propia. 
En la figura 1 se presentan los efectos principales de los factores proporción mango:carambola y temperatura de pasteurización sobre la variable respuesta capacidad antioxidante lipofílica de la mezcla binaria mango:carambola. En la figura 1 también se observa que para ambos factores se logra obtener un nivel máximo de la variable respuesta en niveles aproximadamente intermedios para los rangos evaluados, las curvas tienen forma de parábola, lo que indica adicionalmente que en ambos casos se están obteniendo valores que maximizan la variable respuesta estudiada. Los datos valores que maximizan la capacidad antioxidante lipofílica según el método antes mencionado fueron para el factor mezclas binarias de mango:carambola la proporción de 51:49, y para el factor temperatura de pasteurización el valor de $91^{\circ} \mathrm{C}$.

Figura 1

Efectos principales de los factores proporción mango:carambola y temperatura de pasteurización sobre la capacidad antioxidante lipofílica de la mezcla binaria mango:carambola

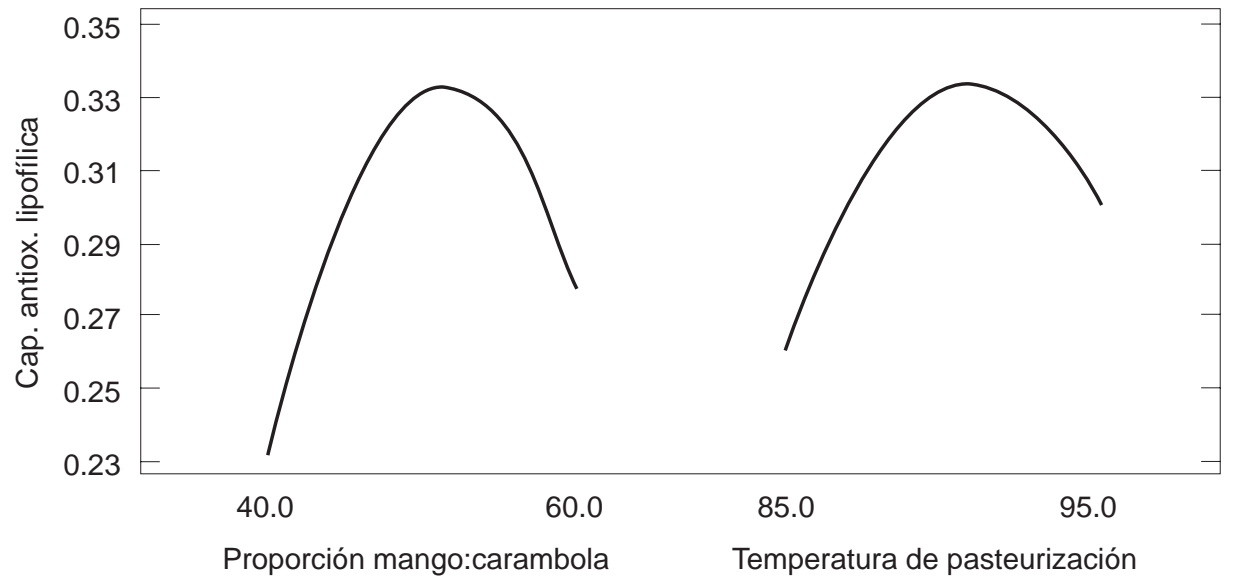

Elaboración propia.

En la figura 2 el diagrama de Pareto estandarizado muestra el efecto significativo evaluado estadísticamente de los factores en estudio, su interacción y efectos cuadráticos sobre la capacidad antioxidante lipofílica, información que se corrobora con el análisis de varianza presentado en el anexo 2. Esa figura indica que tanto los factores principales (proporción de mezclas binarias de mango:carambola y temperatura de 
pasteurización), la interacción de esos factores (AB), así como la tendencia cuadrática de ambos factores (AA y $\mathrm{BB}$ ) son estadísticamente significativos, para un nivel de confianza del 95\% (valor $p$ menor de 0,05 ).

Figura 2

Diagrama de Pareto estandarizado que muestra el efecto significativo evaluado estadísticamente de los factores en estudio, su interacción y efectos cuadráticos sobre la capacidad antioxidante lipofílica

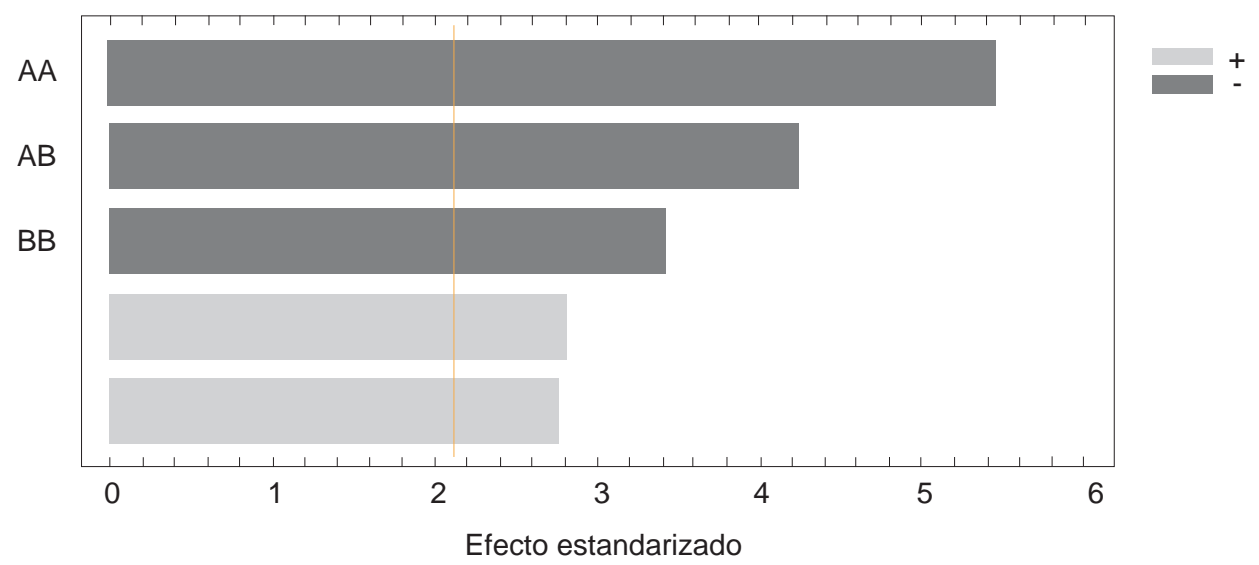

A: Proporción mango:carambola

Elaboración propia.

En la figura 3 se muestran la superficie de respuesta y sus curvas de nivel que maximizan la capacidad antioxidante lipofílica de la mezcla binaria mango:carambola. Esa superficie es considerada forma de «montaña», lo que indica que la variable respuesta presenta un nivel máximo dentro del rango estudiado para cada factor, situación que se corrobora y complementa con lo presentado en la figura 1. 
Figura 3

Superficie de respuesta y curvas de nivel que maximizan la capacidad antioxidante lipofílica de la mezcla binaria mango:carambola

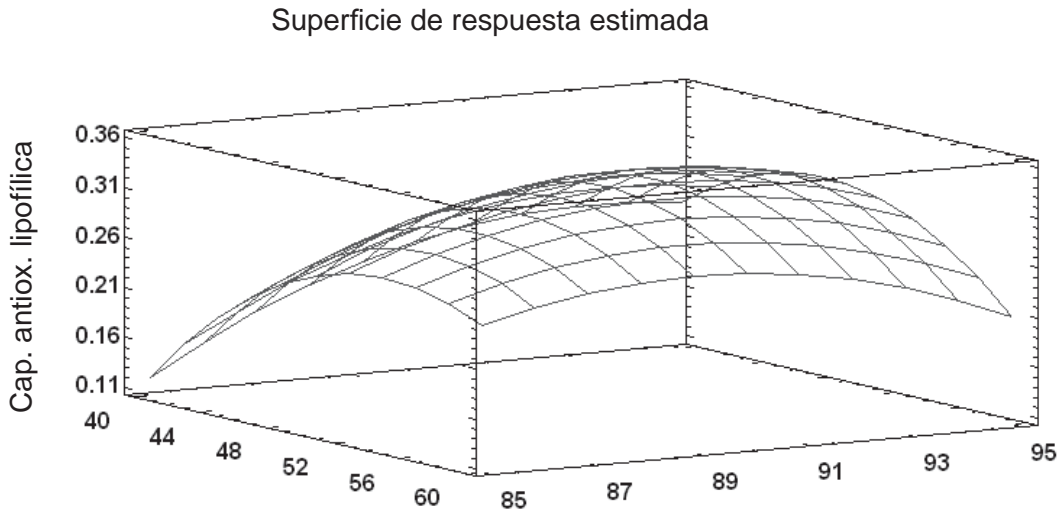

Proporción mango:carambola

Temperatura de pasteurización

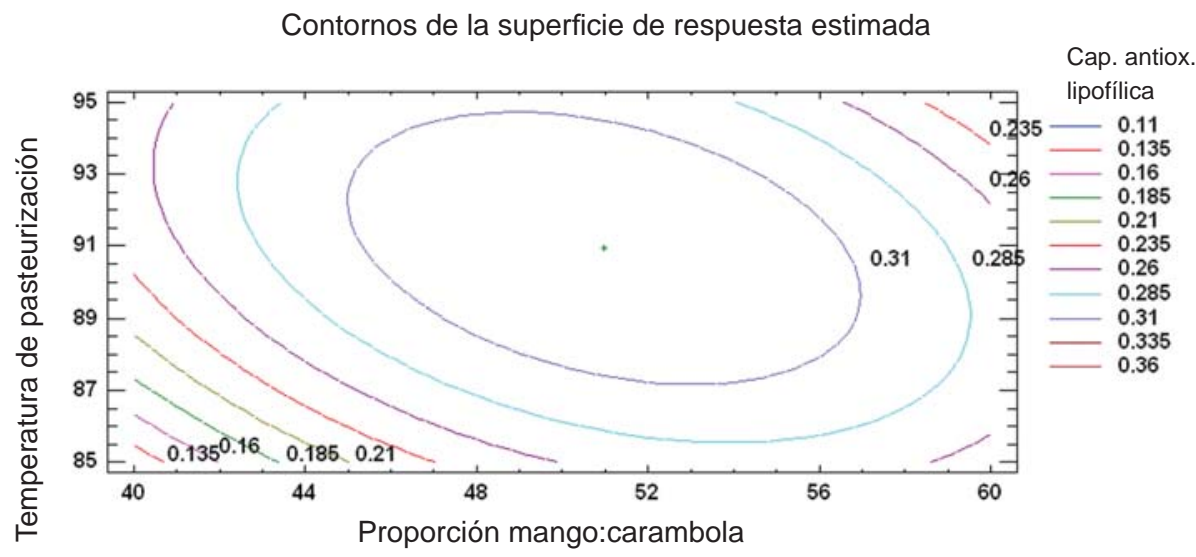

Elaboración propia. 
Cabe mencionar que el valor de maximización teórico obtenido de la capacidad antioxidante lipofílica en esta etapa de Superficie de Respuesta fue de $0,333 \mu \mathrm{mol}$ eq Trolox/g, comparada con el valor experimental obtenido en la siguiente etapa de caracterización del producto final $(0,331 \mu \mathrm{mol}$ eq Trolox/g, valor mostrado en la tabla 8); no se encontraron diferencias estadísticas entre ambas en un nivel de confianza del 95\% (valor p mayor de 0,05), con lo que se estaría completando la validación de la Superficie de Respuesta presentada en la figura 3.

\subsection{Caracterización del producto final}

\subsubsection{Análisis proximal y fisicoquímico}

En la tabla 7 se muestran los resultados del análisis fisicoquímico de la pulpa de mango y carambola que maximizó la capacidad antioxidante lipofílica, determinada en la etapa anterior. Los valores hallados de humedad, cenizas, grasa, proteínas, fibra y carbohidratos fueron de $86,83 \% ; 0,20 \% ; 0,93 \% ; 0,39 \%$ y $11,72 \%$, respectivamente, los que resultan ser valores de mezclas de cada una de las pulpas en forma independiente, además del efecto que tiene la temperatura de pasteurización sobre los alimentos.

Tabla 7

Análisis fisicoquímico de la pulpa de mango:carambola (51:49) pasteurizada $\left(91^{\circ} \mathrm{C}\right)$ que maximizó su capacidad antioxidante lipofílica

\begin{tabular}{lc}
\hline \multicolumn{1}{c}{ Componentes } & Contenido $(\mathbf{g} / \mathbf{1 0 0} \mathbf{g})$ \\
\hline Humedad (\%) & $86,83 \pm 0,04$ \\
Cenizas totales (\%) & $0,32 \pm 0,02$ \\
Grasa (\%) & $0,20 \pm 0,01$ \\
Proteína (\%) & $0,93 \pm 0,03$ \\
Fibra cruda (\%) & $0,39 \pm 0,02$ \\
Carbohidratos (\%) & $11,72 \pm 0,05$ \\
\hline
\end{tabular}

Elaboración propia. 


\subsubsection{Análisis de la capacidad antioxidante lipofílica en el producto final}

En la tabla 8 podemos observar la capacidad antioxidante determinada por el método ABTS. En términos generales, podemos apreciar que la pulpa obtenida tuvo una disminución de su capacidad antioxidante lipofílica en función de las materias primas de la tabla 3. La mencionada disminución de la capacidad antioxidante de las pulpas no solo resulta de las variables tecnológicas aplicadas para la obtención de una pulpa pasteurizada, como son la temperatura de tratamiento térmico o la estandarización del pH y los grados Brix.

Tabla 8

Análisis de la capacidad antioxidante lipofílica de la pulpa de mango:carambola como producto final

\begin{tabular}{lc}
\hline Pulpa & $\begin{array}{c}\text { Capacidad antioxidante lipofílica } \\
(\boldsymbol{\mu m o l ~ e q ~ T r o l o x} / \mathrm{g})\end{array}$ \\
\hline Mango:carambola & $0,331 \pm 0,002$ \\
\hline
\end{tabular}

Elaboración propia.

La disminución de los compuestos bioactivos en las pulpas pasteurizadas se puede deber al efecto que tuvo el tratamiento térmico sobre los compuestos lipofílicos (carotenoides) presentes en el producto, los cuales siguen actuando sinérgicamente, pero con una disminución de su capacidad antioxidante respecto a la materia prima sin procesar. Existe un estudio como el de Encina y Carpio (2009), en el cual la determinación de la capacidad antioxidante en el néctar de tumbo, mediante la metodología del ABTS, permitió la obtención de valores en general que disminuyeron al realizarse el procesamiento de las frutas para obtener dicha bebida, que fue sometida también a tratamiento térmico durante su elaboración, referencias que fueron mencionadas en el estudio realizado por Murillo (2005), citado por (Encina 2006).

\subsubsection{Análisis microbiológico}

En la tabla 9 se muestran los resultados del análisis microbiológico de la mezcla binaria de pulpa de mango y carambola que hace máxima su capacidad antioxidante lipofílica. Como resultado no se detectaron colonias de microorganismos aerobios mesófilos viables ni de hongos 
ni levaduras. De acuerdo con los valores reportados por el Ministerio de Salud (Resolución No 7992 1991), se podría concluir que la mezcla binaria de pulpa de mango y carambola pasteurizada que maximiza la capacidad antioxidante lipofílica elaborada cuenta con condiciones microbiológicas apropiadas para su consumo.

Tabla 9

Carga microbiana de la mezcla binaria de pulpa de mango y carambola pasteurizada que hace máxima su capacidad antioxidante lipofílica

\begin{tabular}{lc}
\hline \multicolumn{1}{c}{ Indicador } & umai.f.c./mI \\
\hline Recuento de aerobios mesófilos viables & $<10$ \\
Recuento de hongos y levaduras & $<10$ \\
\hline
\end{tabular}

Elaboración propia.

\section{CONCLUSIONES}

- El efecto tipo parábola de los factores temperatura de pasteurización (valor $\mathrm{p}=0,0126$ ) y de la proporción de mezclas (valor $\mathrm{p}=0,0110$ ) binarias de pulpa de carambola (Averrhoa carambola) y mango (Mangifera indica L.) sobre la capacidad antioxidante lipofílica fueron estadísticamente significativos (ambos valor p menores de 0,05) para ambos casos, obteniéndose un modelo de Superficie de Respuesta de segundo orden tipo montaña.

- La maximización de la capacidad antioxidante lipofílica aplicando la metodología de Superficie de Respuesta resultó en mezclas binarias de mango:carambola en proporción de 51:49, y para el factor temperatura de pasteurización, el valor de $91{ }^{\circ} \mathrm{C}$, obteniéndose un valor de $0,333 \mu \mathrm{mol}$ eq Trolox/g, dato que se validó con el que se obtuvo en forma experimental $(0,331 \pm 0,002 \mu \mathrm{mol}$ eq Trolox/g), no existiendo diferencias significativas entre ambos valores.

\section{REFERENCIAS}

Alvarado, E. (2011). Estudio del proceso de producción de pulpas de frutas combinadas pasteurizadas y congeladas a mediana escala. (Tesis para obtener el título de ingeniero de alimentos). Guayaquil: Escuela Superior Politécnica del Litoral. 
Amaro, A., Bonilha, P., \& Monteiro, M. (2002). Efeito do tratamento térmico nas características físico-químicas e microbiológicas da polpa de maracujá. Alim. Nutr., 13, 151-162.

Ames, B. N., Shigenaga, M. K., \& Hagen, T. M. (1993). Oxidants, antioxidants, and the degenerative diseases of aging. Procedings of National Academy of Science. Vol. 90.

Andrade-Cuvi, M., Moreno-Guerrero, C., Henriquez-Bucheli, A., Gómez-Gordillo, A., \& Concellon, A. (2010). Influencia de la radiación UV-C como tratamiento poscosecha sobre carambola (Averrhoa carambola) mínimamente procesada almacenada en refrigeración. Revista Iberoamericana de Tecnología Poscosecha. Vol. II, N. ${ }^{\circ} 1,18-27$.

AOAC (1990). Official Methods of Analysis. (15. ${ }^{\mathrm{a}}$ ed.). Washington DC: Association of Official Analytical Chemists.

Aranceta, J., \& Pérez-Rodrigo, C. (2006). Frutas, verduras y salud. Barcelona: Masson.

Baltes, W. (2007). Química de los alimentos. Zaragoza: Acribia.

Brand-Williams, W., Cuvelier, M., \& Berset, C. (1995). Use of free radical method to evaluate antioxidant activity. LebensmittelWissenschaft \& Technologie. Vol. 28. Zurich.

Calzada, J. (1980). Frutales nativos. Lima: Universidad Nacional Agraria La Molina, Facultad de Agronomía.

Castañeda, C., Ramos, Ll., \& Ibáñez, V. (2008). Evaluación de la capacidad antioxidante de siete plantas medicinales peruanas. Revista Horizonte Médico, 1(8).

Encina, Ch. (2005). Determinación de la máxima retención de ácido ascórbico de la conserva de aguaymanto (Physalis peruviana) en almíbar aplicando los métodos Taguchi y Superficie de Respuesta. (Tesis para optar el título de ingeniero en Industrias Alimentarias). Lima: Universidad Nacional Agraria La Molina.

Encina, Ch. (2006). Influencia en el descerado y composición del almibar en la optimización del tratamiento térmico de la conserva de aguaymanto (Physalis peruviana, Linnaeus, 1753). (Tesis para optar al grado de magíster scientiae). Lima: Universidad Nacional Agraria La Molina. 
Encina, Ch., \& Carpio, L. (2011). Determinación de la máxima retención de ácido ascórbico, compuestos bioactivos y capacidad antioxidante en el néctar de tumbo (Passiflora mollisima H. B. K., Bailey). Ingeniería Industrial, 29, 225-245.

ICMSF (2000). Microorganismos de los alimentos, su significado y métodos de numeración. (2 $2^{\text {a }}$ ed., tomo II). Zaragoza: Acribia.

Fellows, P. (2000). Tecnología del procesado de los alimentos: principios y prácticas. Zaragoza: Acribia.

Frazier, W. (1993). Microbiología de los alimentos. Zaragoza: Acribia.

Gómez, O. (2004). Dependencia en la temperatura de los cambios en atributos sensoriales, degradación de ácido ascórbico e inactivación enzimática durante el tratamiento de pasteurización en puré y néctar de tamarindo. (Tesis para optar el grado de magíster). Puebla: Universidad de las Américas Puebla.

Mesa-Vanegas, A., Gaviria, C., Cardona, F., Sáez-Vega, J., Trujillo S., \& Rojano, B. (2010). Actividad antioxidante y contenido de fenoles totales de algunas especies del género Calophyllum. Rev. Cubana Plant. Med.,15(2). La Habana.

Milacatl, V. (2003). Cambios en atributos sensoriales y degradación de ácido ascórbico en función de la temperatura en puré y néctar de mango. (Tesis para optar el título de ingeniero de alimentos). Puebla: Universidad de las Américas Puebla.

Neira, A., \& Yuri, J. (2004). El valor nutritivo de la fruta. Boletín Técnico 4(4). Talca: Universidad de Talca.

Osorio, O. (2008). Influencia de tratamientos térmicos en la calidad y estabilidad del puré de fresa (Fragaria x ananassa, cv Camarosa). (Tesis para optar el grado de doctor). Valencia: Universidad Politécnica de Valencia.

Ottone, G. (2001). Procesamiento y exportación de frutas tropicales. (Tesis para optar el título de ingeniero en Industrias Alimentarias). Lima: Universidad Nacional Agraria La Molina.

Re, R., Pellegrini, N., Proteggente, A., Pannala, A., Yang, M., \& RiceEvans, C. (1999). Antioxidant activity applying an improved ABTS radical cation decolorization assay. Free radical Biology and Medicine, 26. 
Rees, T. A., \& Bettison, J. (1994). Procesado térmico y envasado de alimentos. Zaragoza: Acribia.

Resolución No 7992 (1991). Elaboración, conservación y comercialización de jugos, concentrados, néctares, pulpas, pulpas edulcoradas y refrescos de frutas. Bogotá: Ministerio de Salud.

Reyes, M., Gómez-Sánchez, I., Espinoza, C., Bravo, F., \& Ganoza, L. (2009). Tabla peruana de composición de alimentos. (8. ${ }^{\mathrm{a}}$ ed.). Lima: Ministerio de Salud, Instituto Nacional de Salud.

StatPoint, Inc. (2013). Statgraphics Centurion XVI. Copia de evaluación de software estadístico. Herndon, VA. 


\section{Anexo 1 \\ Cálculos para la determinación del tiempo de pasteurización para las temperaturas de 85,90 y $95^{\circ} \mathrm{C}$}

Para la determinación del tiempo de pasteurización se determinaron las variables de la siguiente ecuación:

$$
U P_{0}=P_{0}=D_{0} \times(\log a-\log b)
$$

Donde:

a: la Población inicial se determinó por triplicado en la mezcla binaria que presentó mayor $\mathrm{pH}$. Siendo de $1,6 \times 10^{4} \mathrm{ufc} / \mathrm{ml}$

b: la Población final debía ser menor a 10 ufc/ml

El tiempo de reducción decimal (D) se obtuvo de la literatura, a la temperatura de referencia de $85^{\circ} \mathrm{C}: \mathrm{D}_{85^{\circ} \mathrm{C}}=5$ minutos

La constante de resistencia térmica $(Z)$ establecida por la literatura: $Z=5,5^{\circ} \mathrm{C}$

Se determinó que el tiempo requerido para destruir un número específico de microorganismos a $85^{\circ} \mathrm{C}\left(\mathrm{P}_{\mathrm{o}}\right)$ fue de $16,02 \mathrm{~min}$.

Para el cálculo del tiempo de tratamiento térmico equivalente para las temperaturas de 85,90 y $95^{\circ} \mathrm{C}$ se usó la siguiente ecuación:

$$
P_{x}=P_{0} \times 10^{((T 0-T x) / z))}
$$

Donde:

$\mathrm{P}_{\mathrm{o}}=$ tiempo requerido para destruir un número específico de microorganismos a 85 ${ }^{\circ} \mathrm{C}$

$\mathrm{T}_{\mathrm{o}}=$ temperatura de referencia, $85^{\circ} \mathrm{C}$

$\mathrm{T}_{\mathrm{x}}=$ temperatura a evaluar en el medio de calentamiento para realizar la pasteurización

Z = 5,5 ${ }^{\circ} \mathrm{C}$; parámetro cinético del Bysosschlamys fulva 
René Encina, Andrea Bernal, Daniel Rojas

Análisis de varianza para la capacidad antioxidante lipofílica

\begin{tabular}{cc}
\hline Temperatura & $\begin{array}{c}\text { Tiempo de muerte térmica } \\
(\mathrm{min})\end{array}$ \\
\hline $85^{\circ} \mathrm{C}$ & 16,02 \\
$90^{\circ} \mathrm{C}$ & 1,98 \\
$95^{\circ} \mathrm{C}$ & 0,24 \\
\hline
\end{tabular}


Anexo 2

Análisis de varianza para la capacidad antioxidante lipofílica

\begin{tabular}{llllll}
\hline Fuente & $\begin{array}{l}\text { Suma de } \\
\text { cuadrados }\end{array}$ & GI & Cuadrado medio & Razón-F & Valor-P \\
\hline $\begin{array}{l}\text { A: Proporción } \\
\text { mango:carambola }\end{array}$ & 0.00919368 & 1 & 0.00919368 & 7.93 & 0.0110 \\
$\begin{array}{l}\text { B: Temperatura de } \\
\text { pasteurización }\end{array}$ & 0.00879138 & 1 & 0.00879138 & 7.58 & 0.0126 \\
AA & 0.03460535185 & 1 & 0.03460535185 & 29.84 & 0.0000 \\
AB & 0.02076672 & 1 & 0.02076672 & 17.91 & 0.0005 \\
BB & 0.01365810074 & 1 & 0.01365810074 & 11.78 & 0.0028 \\
bloques & 0.00002225851852 & 2 & 0.00001112925926 & 0.01 & 0.9905 \\
Error total & 0.02203494519 & 19 & 0.001159733957 & & \\
Total (corr.) & 0.1090724363 & 26 & & & \\
\hline
\end{tabular}

R-cuadrada $=79.79787934$ por ciento

R-cuadrada (ajustada por g.l.) $=72.35499278$ por ciento

Error estándar del est. $=0.03405486686$

Error absoluto medio $=0.02420576132$

Estadístico Durbin-Watson = $3.413462402(\mathrm{P}=0.9996)$

Autocorrelación residual de Lag $1=-0.7270257974$

\section{El StatAdvisor}

La tabla ANOVA particiona la variabilidad de capacidad antioxidante lipofílica en piezas separadas para cada uno de los efectos. Entonces prueba la significancia estadística de cada efecto comparando su cuadrado medio contra un estimado del error experimental. En este caso, 5 efectos tienen una valor-P menor que 0.05 , indicando que son significativamente diferentes de cero con un nivel de confianza del 95.0\%.

El estadístico R-Cuadrada indica que el modelo, así ajustado, explica $79.79787934 \%$ de la variabilidad en capacidad antioxidante lipofílica. El estadístico R-cuadrada ajustada, que es más adecuado para comparar modelos con diferente número de variables independientes, es $72.35499278 \%$. El error estándar del estimado muestra que la desviación estándar de los residuos es 0.03405486686 . El error medio absoluto (MAE) de 0.02420576132 es el valor promedio de los residuos. El estadístico de Durbin-Watson (DW) prueba los residuos para determinar si hay alguna correlación significativa basada en el orden en que se presentan los datos en el archivo. Puesto que el valor-P es mayor que 5.0\%, no hay indicación de autocorrelación serial en los residuos con un nivel de significancia del $5.0 \%$. 